\title{
Femtosecond Laser Writing of Optical-Lattice-Like Cladding Structures for Three-Dimensional Waveguide Beam Splitters in $\mathrm{LiNbO}_{3}$ Crystal
}

\author{
Jinman Lv, Yazhou Cheng, Javier R. Vázquez de Aldana, Xiaotao Hao, and Feng Chen, Senior Member, OSA
}

\begin{abstract}
The waveguide beamsplitters with diverse configurations in $\mathrm{LiNbO}_{3}$ crystal have been produced by direct femtosecond laser writing of a family of optical-lattice-like cladding structures. By on demand design of the lattice tracks with "defect" lines, the efficient beam guiding and tailoring have been implemented in the structures. With a family of three-element integration of structures, three-dimensional (3-D) $1 \times 3$ beamsplitting at the telecommunication wavelength of $1550 \mathrm{~nm}$ was realized. Different from the Type I modification of $\mathrm{LiNbO}_{3}$ waveguides, the guiding cores of the optical-lattice-like cladding waveguide structures we fabricated locate in regions that are surrounded by the laser-induced-tracks. This paper opens the alternative way to construct complex integrated platforms in $\mathrm{LiNbO}_{3}$ crystal by using femtosecond laser writing.
\end{abstract}

Index Terms-Beam splitters, laser materials processing, lithium niobate, waveguides.

\section{INTRODUCTION}

A $\mathrm{S}$ the basic components in integrated photonics, optical waveguides confine the light propagation in very small volumes with dimensions of several micrometers, achieving relatively higher optical intensities with respect to the bulk materials [1]. Photonic devices including switchers, routers, demultiplexers, and modulators are widely used in optical telecommunication systems [2]. Waveguide beam splitters, which can distribute signals from one input port to two or more output ports, are fundamental elements in photonics applications for signal routing and signal processing.

Several techniques, such as metal-ion indiffusion, ion/proton exchange, ion implantation/irradiation and femtosecond (fs) laser micromachining, have already been used to fabricate optical waveguides in a wide range of optical materials [3]-[9]. Owing to the capability for $3 \mathrm{D}$ processing, the femtosecond laser writing has emerged as a powerful and promising method to fabricate optical waveguides in various transparent materials since the pioneering work of Davis et al., in 1996 [10]-

Manuscript received February 02, 2016; revised May 05, 2016; accepted May 25, 2016. Date of publication May 26, 2016; date of current version July 21, 2016. The work was supported by National Natural Science Foundation of China under Grant 11274203, and Ministerio de Economía y Competitividad (Project FIS2013-44174-P), Spain.

J. Lv, Y. Cheng, X. Hao, and F. Chen are with the School of Physics, State Key Laboratory of Crystal Materials, Shandong University, Jinan 250100, China (e-mail: ljm@sdu.edu.cn; chengyazhou@ sdu.edu.cn; haoxt@ sdu.edu.cn; drfchen@sdu.edu.cn).

J. R. Vázquez de Aldana is with the Laser Microprocessing Group, Universidad de Salamanca, Salamanca 37008, Spain (e-mail: jrval@usal.es).

Color versions of one or more of the figures in this paper are available online at http://ieeexplore.ieee.org.

Digital Object Identifier 10.1109/JLT.2016.2573841

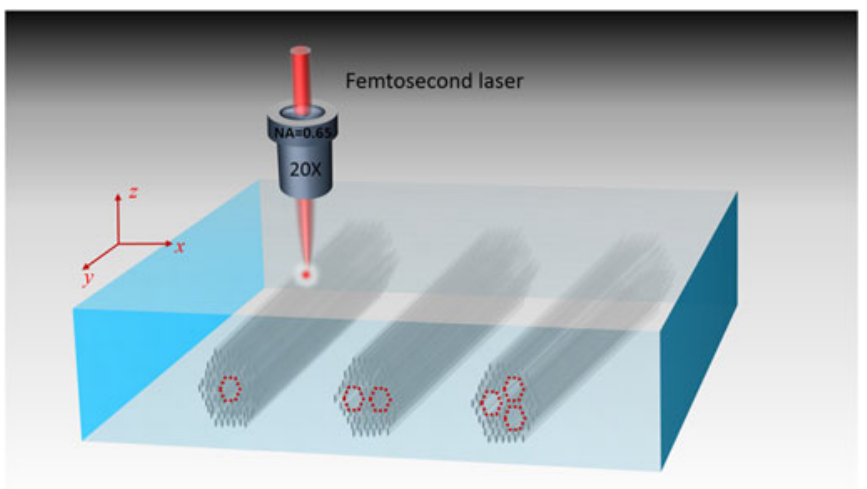

Fig. 1. The schematic fs-laser-writing process of the $\mathrm{LiNbO}_{3}$ waveguide beam splitters.

[13]. Depending on the parameters of the fs-laser pulses and the nature of the materials, the refractive index change $(\Delta n)$ induced in the fs-laser-irradiated region may be positive or negative, referring to Type I and II modifications [14], [15]. As a result, typical waveguide geometries (single-line, dual-line and depressed claddings) have been developed accordingly in a number of crystals, which show diverse guiding properties and bulk-related features for versatile applications [8].

Lithium niobate $\left(\mathrm{LiNbO}_{3}\right)$ is one of the most favorite crystals in integrated optics owing to its outstanding features of electro-optic, acousto-optic, piezoelectric and nonlinear optical properties [16]. It has been widely used for active optical applications as electro-optic modulators, surface-acoustic wave devices, holographic storage, and frequency converters [17]-[19]. Femtosecond laser writing of $\mathrm{LiNbO}_{3}$ waveguides has been implemented with diverse configurations [20]-[24]. In previous work, we have fabricated 3D waveguide beam splitters based on Type I modification of $n_{\mathrm{e}}$ in $\mathrm{LiNbO}_{3}$ crystal by laser writing [25]. In this work, we report on the fabrication of $3 \mathrm{D}$ waveguide beam splitters in $\mathrm{LiNbO}_{3}$ crystal based on the Type II modification of $n_{\mathrm{o}}$ through so-called optical-lattice-like geometries, which was first applied to Nd:YAG crystal for guided beam tailoring [26]. The guiding properties of the waveguides are investigated at the telecommunication wavelength of $1550 \mathrm{~nm}$.

\section{EXPERIMENTS IN DETAILS}

Fig. 1 depicts the schematic fs-laser-writing process of the $\mathrm{LiNbO}_{3}$ waveguide beam splitters. The $z$-cut $\mathrm{LiNbO}_{3}$ crystal 


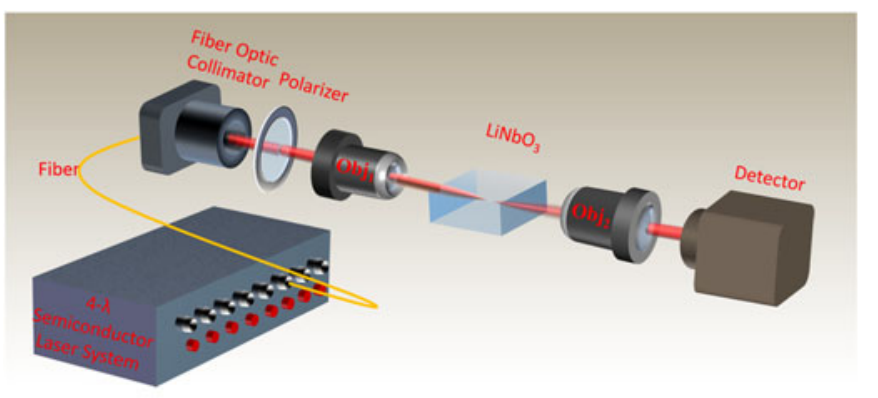

Fig. 2. The schematic end-face coupling arrangement for investigation of the $\mathrm{LiNbO}_{3}$ waveguide beam splitters at $1550 \mathrm{~nm}$.

used in this work was cut with dimensions of $10(x) \times 10(y) \times$ $1(z) \mathrm{mm}^{3}$ and was optically polished. The waveguide beam splitters of optical-lattice-like cladding structures were fabricated by using the laser facility of Universidad de Salamanca, Spain. A Ti:Sapphire regenerative amplifier (Spitfire, Spectra Physics) was utilized as a laser source to generate linearly polarized pulses (central wavelength of $795 \mathrm{~nm}$, pulse duration of $120 \mathrm{fs}$, repetition rate of $1 \mathrm{kHz}$ and maximum pulse energy of $50 \mu \mathrm{J})$. The incident fs laser pulse energy was set to $2 \mu \mathrm{J}$ with a calibrated neutral density filter, a half-wave plate and a linear polarizer, and then was focused by a $20 \times$ microscope objective (N.A. $=0.65$ ) at a maximum depth of $180 \mu \mathrm{m}$ beneath the upper surface $\left(10 \times 10 \mathrm{~mm}^{2}\right)$ of the sample. During the irradiation process, the sample was placed at a micro-positioning $X-Y-Z$ motorized stage and was scanned at a constant velocity of $750 \mu \mathrm{m} / \mathrm{s}$ along the $y$-axis, producing a damage track inside the sample. The procedure was performed at different depths of the sample with a lateral separation of $10 \mu \mathrm{m}$ between each two adjacent tracks. With these conditions, 3-D waveguide beam splitters (with a guiding core surrounded by arrayed damage tracks, i.e., optical-lattice-like geometries) were fabricated in $\mathrm{LiNbO}_{3}$ crystal. As a result, a $1 \times 1$ waveguide beam splitter WG1, a $1 \times 2$ waveguide beam splitter WG2 and a $1 \times 3$ waveguide beam splitter WG3 have been fabricated in $\mathrm{LiNbO}_{3}$ crystal.

A microscope (Axio Imager, Carl Zeiss) was utilized to photograph the cross sections of the waveguide beam splitters in $\mathrm{LiNbO}_{3}$ crystal. An end-face coupling arrangement, as shown in Fig. 2, was applied to experimentally characterize the nearfield modal profiles of the waveguide beam splitters. The incident light beam at $1550 \mathrm{~nm}$, which was generated in the $4-\lambda$ semiconductor laser system (GCSLS-O, China Daheng Group, Inc.), was coupled into a fiber cable. By using the fiber optic collimator (GCX-L, China Daheng Group, Inc.), the divergent light was collimated. Afterwards, a pair of MIR microscope objective lens $(\mathrm{ZnSe}$, LFO-5-12-3.75, N.A. $=0.13)$ were used to couple the linearly polarized light (TE or TM mode) into and out of the waveguide beam splitter, and finally a CCD camera was employed to observe and record the near-field intensity distributions at $1550 \mathrm{~nm}$. In order to determine the propagation losses of the waveguide beam splitter, a power meter was utilized to measure the powers from the input and output end-faces.

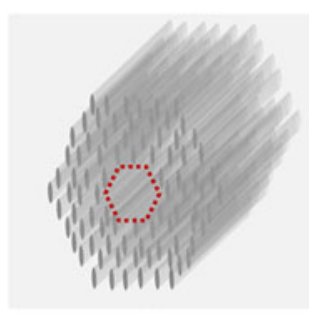

(a)

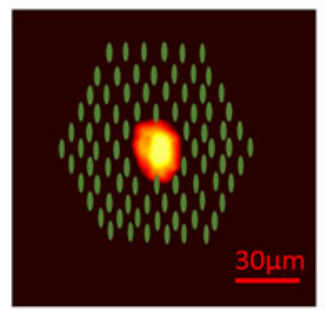

(c)

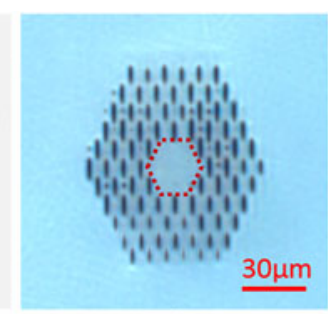

(b)

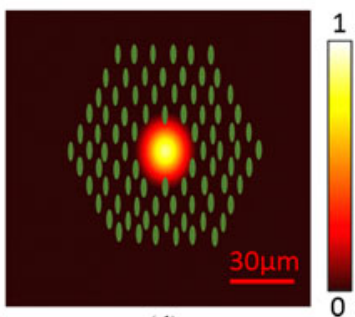

(d)
Fig. 3. (a) The cross-sectional sketch (b) microscopic image of the cross section (c) measured near-field modal profile along TE polarization at $1550 \mathrm{~nm}$ and (d) simulated modal profile of the $1 \times 1$ waveguide beam splitter WG1.

\section{RESUlTS AND DISCUSSION}

Fig. 3(a) shows the cross-sectional sketch of the $1 \times 1$ waveguide beam splitter WG1 which was defined as Element 1 . Fig. 3(b) depicts the microscopic image of the cross section of the Element 1 in $\mathrm{LiNbO}_{3}$ crystal. The transverse length of the fs-laser inscribed tracks is $10 \mu \mathrm{m}$ and the separation between two adjacent tracks is $10 \mu \mathrm{m}$. The optical-lattice-like structure of the Element 1 has a length of $10 \mathrm{~mm}$ and a guiding core with a quasi-hexagonal cross-section $\left(\sim 30 \times 30 \mu \mathrm{m}^{2}\right)$. In order to obtain an approximate value of the maximum refractive index contrast of the waveguide, we measured the N.A. of the waveguide and used the method reported in [27]. Considering the combination of the damage induced refractive index reduction and the stress induced refractive index increment, the maximum refractive index change $\Delta n_{\mathrm{o}}$ of the Element 1 was estimated to be $-5 \times 10^{-3}$ for the TE mode at $1550 \mathrm{~nm}$. Fig. 3(c) shows the measured near-field intensity distribution of Element 1 for the TE mode at $1550 \mathrm{~nm}$. Based on the reconstructed refractive index distribution, we simulated near-field modal profile of Element 1 for the TE mode at $1550 \mathrm{~nm}$ by using the software Rsoft $₫$ Beam-Prop [28] through Finite Difference Beam Propagation Method (FD-BPM) [29], as shown in Fig. 3(d). As one can see, the calculated near-field modal profile of Element 1 is basically in agreement with the experimental results.

As the light propagates along the central core of Element 1 , we introduced additional damage tracks as "defect lines" in the core region of Element 1 to form Elements 2 and 3, which modify the light propagation to tailor the light intensity distribution. Fig. 4(a) depicts the prototype of $1 \times 2$ beam splitter WG2 which is connected by Elements 1, 2 and 3 in sequence, including the schematic sketches (top) and microscopic photographs (below) of Elements 1, 2 and 3, respectively. As we can see, Elements 1, 2 and 3 have the lengths of $2 \mathrm{~mm}, 4 \mathrm{~mm}$ and $4 \mathrm{~mm}$, respectively. Fig. 4(b) shows the measured near-field 


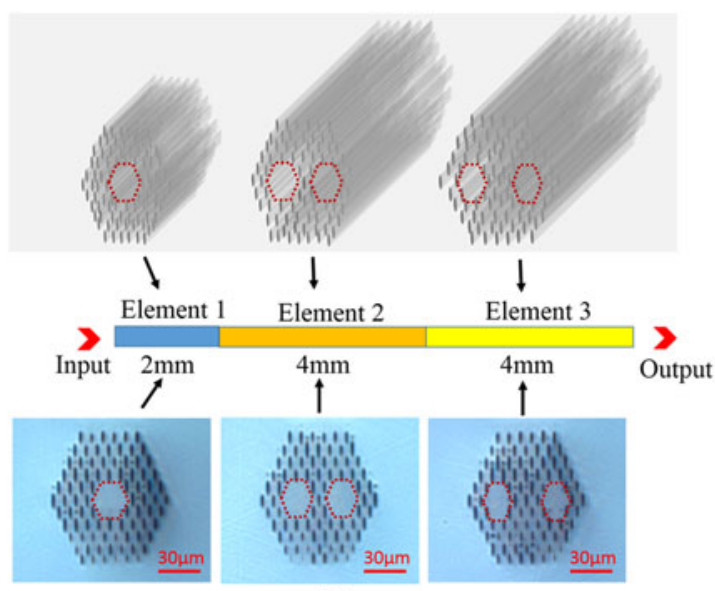

(a)

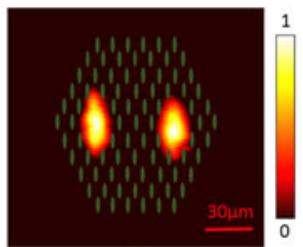

(b)

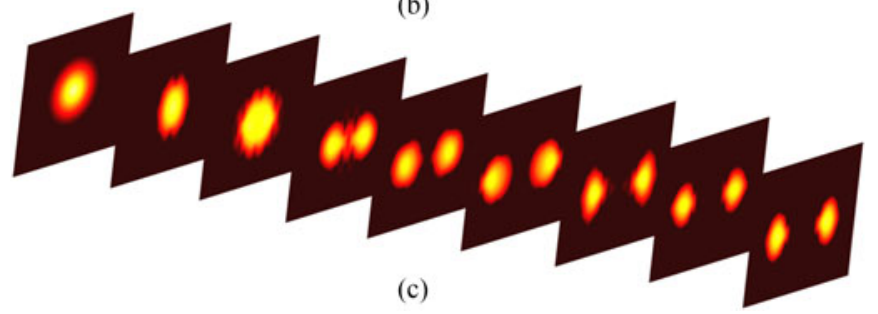

Fig. 4. (a) The prototype of $1 \times 2$ beam splitter WG2 connected by Elements 1, 2 and 3 in sequence, including the cross-sectional sketches (top) and microscopic images (below) of the elements. (b) The measured near-field modal profile along TE polarization at $1550 \mathrm{~nm}$ and (c) simulated modal profile evolution of the $1 \times 2$ waveguide beam splitter WG2.

intensity distribution from the output of the WG2 for the TE mode at $1550 \mathrm{~nm}$, exhibiting a clear beam-profile splitting with a splitting ratio of 0.50:0.50 from the two arms. It indicates that the input light beam propagating through the $1 \times 2$ beam splitter is equally divided into two parts and guided out, showing effective performance for a Y-branch-like function. Fig. 4(c) depicts the simulated near-field beam profile evolution of $1 \times 2$ beam splitter for the TE mode at $1550 \mathrm{~nm}$, which matches well with the experimental result.

Similarly, we constructed $1 \times 3$ beam splitter WG3 by combining Elements 1, 4 and 5 in a joint structure. Fig. 5(a) shows the prototype of $1 \times 3$ beam splitter, including the schematic sketches (top) and microscopic photographs (below) of the Element 1, 4 and 5, respectively. As we can see, Elements 1, 4 and 5 have the lengths of $2 \mathrm{~mm}, 4 \mathrm{~mm}$ and 4 $\mathrm{mm}$, respectively. Fig. 5(b) shows the experimental near-field intensity distribution from the output of the $1 \times 3$ beam splitter for the TE mode at $1550 \mathrm{~nm}$ with a measured intensity splitting ratio of 0.50:0.49:0.51 for the three arms. This result indicates that our fabricated $1 \times 3$ beam splitter possesses satisfactory performance with quasi-equal output light powers and we can

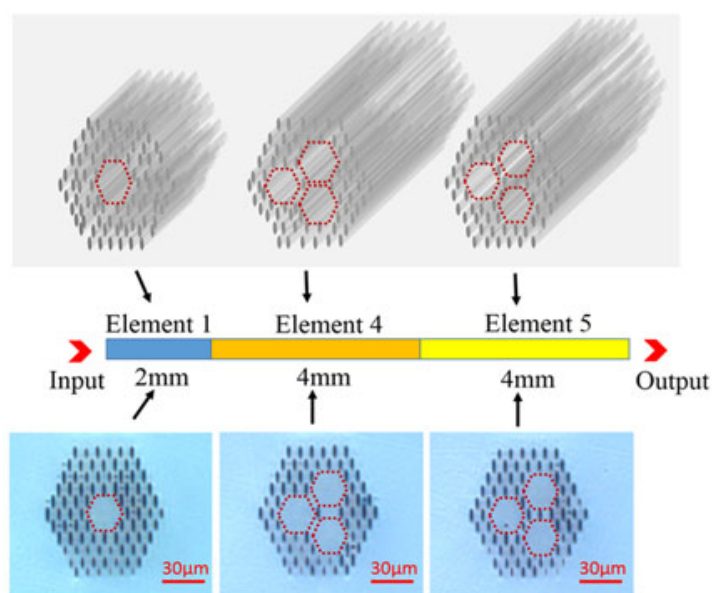

(a)

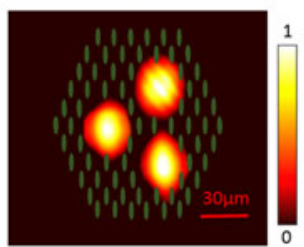

(b)

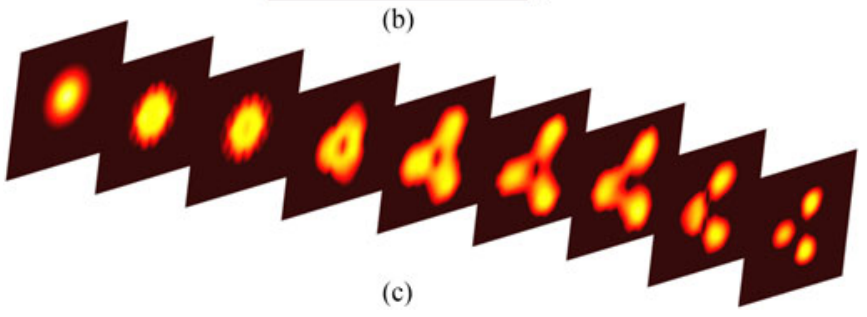

Fig. 5. (a) The prototype of $1 \times 3$ beam splitter WG3 connected by Elements 1,4 and 5 in sequence, including the cross-sectional sketches (top) and microscopic images (below) of the elements. (b) The measured near-field modal profile along TE polarization at $1550 \mathrm{~nm}$ and (c) simulated modal profile evolution of the $1 \times 3$ waveguide beam splitter WG3.

further improve the splitting ratio by optimizing the processing parameters. The simulated near-field beam profile evolution of $1 \times 3$ beam splitter for the TE mode at $1550 \mathrm{~nm}$, as shown in Fig. 5(c), accords with the experimental result.

Fig. 6 shows the all-angle light transmission of the WGs 13 to investigate the thorough information of the polarization effects of the guidance at $1550 \mathrm{~nm}$ wavelength. It is found that as the light polarization angle changes the output light power varies, showing the light guidance is polarization-dependent. Obviously, there is no guidance along the TM polarization $\left(n_{\mathrm{e}}\right)$, and the optimum guidance is realized along the TE polarization $\left(n_{\mathrm{o}}\right)$. This is different from the Type I waveguide beam splitters in $\mathrm{LiNbO}_{3}$ (i.e., guided at $n_{\mathrm{e}}$ only) [25].

The total losses of the waveguide beam splitters (including propagation losses and coupling losses) were measured by using the end-face coupling system at $1550 \mathrm{~nm}$. The coupling loss was estimated to be $0.7 \mathrm{~dB}$ by considering the overlap of the profiles of the incident light beam and waveguide modes. Table I shows the propagation losses of the WGs 1-3 along TE polarization at $1550 \mathrm{~nm}$. Please note that these values were obtained as sum propagation losses (i.e., the bending loss of 


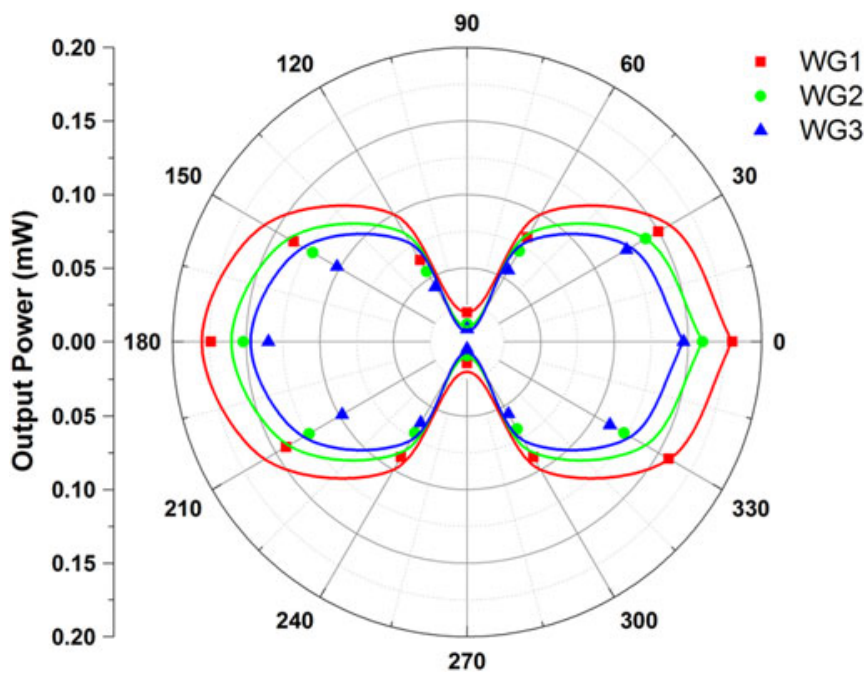

Fig. 6. The Polar images of the output light power of WG1 (red squares), WG2 (green circles) and WG3 (blue triangles) and the corresponding fits (lines) at $1550 \mathrm{~nm}$.

TABLE I

The Propagation Losses of Laser-Written WaVeguide Beam SplitTers ALONG TE POLARIZATION AT 1550 NM

\begin{tabular}{lc}
\hline \hline Waveguide configurations & Propagation loss $(\mathrm{dB} / \mathrm{cm})$ \\
\hline WG1 & 1.27 \\
WG2 & 1.63 \\
WG3 & 2.11 \\
\hline \hline
\end{tabular}

the structures were included as well). It was clear that, as the number of output arm increases the propagation loss of the beam splitter becomes larger, which may be partly attributed to the imperfections of the multi-arm structures. Further reduction of the propagation loss may be realized by increasing the guiding core volume and amount of the track lattice layers of the waveguide beam splitter [26], [30].

\section{CONCLUSION}

We have designed and fabricated 3D waveguide beam splitters supporting guidance along TE $\left(n_{\mathrm{o}}\right)$ polarization at $1550 \mathrm{~nm}$ in $\mathrm{LiNbO}_{3}$ crystal by direct femtosecond laser writing. The near-field modal profiles of the waveguide beam splitters are experimentally and numerically investigated, and the simulations are in good agreement with the experimental results. The propagation loss of the $1 \times 3$ beam splitter is $\sim 2.1 \mathrm{~dB} / \mathrm{cm}$ and the beam splitting ratio is approximately equal, indicating our approach to 3D waveguide beam splitters for potential application as integrated devices (e.g., electrooptic modulators) based on $\mathrm{LiNbO}_{3}$ crystals.

\section{REFERENCES}

[1] G. C. Righini and A. Chiappini, "Glass optical waveguides: A review of fabrication techniques," Opt. Eng., vol. 54, no. 7, Jul. 2014, Art. no. 071819.
[2] F. Qiu, A. M. Spring, F. Yu, I. Aoki, A. Otomo, and S. Yokoyama, "Thin $\mathrm{TiO}_{2}$ core and electro-optic polymer cladding waveguide modulators," Appl. Phys. Lett., vol. 102, no. 23, Jun. 2013, Art. no. 233504.

[3] M. Quintanilla, E. M. Rodríguez, E. Cantelar, F. Cussó, and C. Domingo, "Micro-Raman characterization of $\mathrm{Zn}$-diffused channel waveguides in $\mathrm{Tm}^{3+}: \mathrm{LiNbO}_{3}, "$ Opt. Express, vol. 18, no. 6, pp. 5449-5458, Mar. 2010.

[4] A. Tervonen, B. R. West, and S. Honkanen, "Ion-exchanged glass waveguide technology: A review," Opt. Eng., vol. 50, no. 7, Jul. 2011, Art. no. 071107.

[5] F. Chen, "Micro- and submicrometric waveguiding structures in optical crystals produced by ion beams for photonic applications," Laser Photon. Rev., vol. 6, no. 5, pp. 622-640, Sep. 2012.

[6] Y. Tan, Q. Luan, F. Liu, S. Akhmadaliev, S. Zhou, and F. Chen, "Swift carbon ion irradiated Nd:YAG ceramic optical waveguide amplifier," Opt. Express, vol. 21, no. 12, pp. 13992-13997, Jun. 2013.

[7] M. Ams, P. Dekker, G. D. Marshall, and M. J. Withford, "Monolithic $100 \mathrm{~mW} \mathrm{Yb}$ waveguide laser fabricated using the femtosecond-laser direct-write technique," Opt. Lett., vol. 34, no. 3, pp. 247-249, Feb. 2009.

[8] F. Chen and J. R. Vazquez de Aldana, "Optical waveguides in crystalline dielectric materials produced by femtosecond-laser micromachining," Laser Photon. Rev., vol. 8, no. 2, pp. 251-275, May. 2014.

[9] D. Choudhury, J. R. Macdonald, and A. K. Kar, "Ultrafast laser inscription: Perspectives on future integrated applications," Laser Photon. Rev., vol. 8, no. 6, pp. 827-846, Nov. 2014.

[10] S. Nolte, M. Will, J. Burghoff, and A. Tuennermann, "Femtosecond waveguide writing: a new avenue to three dimensional integrated optics," Appl. Phys. A, vol. 77, no. 1, pp. 109-111, Mar. 2003.

[11] R. R. Gattass and E. Mazur, "Femtosecond laser micromachining in transparent materials," Nature Photon., vol. 2, no. 4, pp. 219-225, Apr. 2008.

[12] K. M. Davis, K. Miura, N. Suguimoto, and K. Hirao, "Writing waveguides in glass with a femtosecond laser," Opt. Lett., vol. 21, no. 21, pp. 17291731, Nov. 1996.

[13] M. Ams, G. D. Marshall, P. Dekker, J. A. Piper, and M. J. Withford, "Ultrafast laser written active devices," Laser Photon. Rev., vol. 3, no. 6, pp. 535-544, Nov. 2009.

[14] J. Burghoff, S. Nolte, and A. Tünnermann, "Origins of waveguiding in femtosecond laser-structured $\mathrm{LiNbO}_{3}$," Appl. Phys. A., vol. 89, no. 1, pp. 127-132, Oct. 2007.

[15] S. Gross, M. Dubov, and M. J. Withford, "On the use of the Type I and II scheme for classifying ultrafast laser direct-write photonics," Opt. Express, vol. 23, no. 6, pp. 7767-7770, Mar. 2015.

[16] L. Arizmendi, "Photonic applications of lithium niobate crystals," Phys. Status Solidi A, vol. 201, no. 2, pp. 253-283, Jan. 2004.

[17] K. Buse, A. Adibi, and D. Psaltis, "Non-volatile holographic storage in doubly doped lithium niobate crystals," Nature, vol. 393, no. 6686, pp. 665-668, Jun. 1998.

[18] W. Sohler et al., "Integrated optical devices in lithium niobate," Opt. Photon. News, vol. 19, no. 1, pp. 24-31, Jan. 2008.

[19] E. L. Wooten et al., "A review of lithium niobate modulators for fiberoptic communication systems," IEEE J. Sel. Top. Quantum Electron., vol. 6, no. 1, pp. 69-82, Jan./Feb. 2000.

[20] Y. Liao et al., "Electro-optic integration of embedded electrodes and waveguides in $\mathrm{LiNbO}_{3}$ using a femtosecond laser," Opt. Lett., vol. 33, pp. 2281-2283, Oct. 2008.

[21] J. Thomas et al., "Laser direct writing: Enabling monolithic and hybrid integrated solutions on the lithium niobate platform," Phys. Status Solidi A, vol. 208, no. 2, pp. 276-283, Feb. 2011.

[22] H. T. Bookey et al., "Femtosecond laser inscription of low insertion loss waveguides in z-cut lithium niobate," IEEE Photon. Technol. Lett., vol. 19, no. 9-12, pp. 892-894, May./Jun. 2007.

[23] R. He et al., "Femtosecond laser micromachining of lithium niobate depressed cladding waveguides," Opt. Mater. Express, vol. 3, no. 9, pp. 1378-1384, Aug. 2013.

[24] S. Kroesen, W. Horn, J. Imbrock, and C. Denz, "Electro-optical tunable waveguide embedded multiscan Bragg gratings in lithium niobate by direct femtosecond laser writing," Opt. Express, vol. 22, no. 19, pp. 23339 23348, Sep. 2014.

[25] J. M. Lv, Y. Z. Cheng, W. H. Yuan, X. T. Hao, and F. Chen, "Threedimensional femtosecond laser fabrication of waveguide beam splitters in $\mathrm{LiNbO}_{3}$ crystal," Opt. Mater. Express, vol. 5, no. 6, pp. 1274-1280, May 2015.

[26] Y. Jia et al., "Monolithic crystalline cladding microstructures for efficient light guiding and beam manipulation in passive and active regimes," Sci. Rep., vol. 4, p. 5598, Aug. 2014. 
[27] J. Siebenmorgen, K. Petermann, G. Huber, K. Rademaker, S. Nolte, and A. Tünnermann, "Femtosecond laser written stress-induced $\mathrm{Nd}: \mathrm{Y}_{3} \mathrm{Al}_{5} \mathrm{O}_{12}$ (Nd:YAG) channel waveguide laser," Appl. Phys. B, vol. 97, no. 2, pp. 251-255, Oct. 2009.

[28] Rsoft Design Group. Computer software beamPROP version 8. (2007). [Online]. Available: http://www.rsoftdesign.com.

[29] D. Yevick and W. Bardyszewski, "Correspondence of variational finitedifference (relaxation) and imaginary-distance propagation methods for modal analysis," Opt. Lett., vol. 17, no. 5, pp. 329-330, Mar. 1992.

[30] A. Okhrimchuk, V. Mezentsev, A. Shestakov, and I. Bennion, "Low loss depressed cladding waveguide inscribed in YAG:Nd single crystal by femtosecond laser pulses," Opt. Express, vol. 20, no. 4, pp. 3832-3843, Feb. 2012.

Jinman $\mathbf{L v}$ received the B.A. degree from Shandong University, Jinan, China, in 2013, where she is currently working toward the Ph.D. degree. Her research interests include fabrication of optical waveguides in optical materials by using ion beam implantation and femtosecond laser inscription technique.

Yazhou Cheng received the B.S. degree form Shandong University, Shandong, China, in 2007, and the M.S. degree from the Royal Institute of Technology, Sweden, in 2009. He is currently working toward the Ph.D. degree at Shandong University, Shandong, China. His research interests include optical waveguides fabricated by ion beam implantation and femtosecond laser inscription.
Javier R. Vázquez de Aldana received the Bachelor of Science degree in 1997, and the Ph.D. degree from the University of Salamanca, Salamanca, Spain, in 2001. He is currently an Associate Professor at the Science Faculty, University of Salamanca, Salamanca, Spain. He is a Member of the Laser Microprocessing Research Group and also a Technical and Scientific Advisor of the Laser Facility, University of Salamanca. His research interests include the interaction of intense femtosecond pulses with materials and its application to fabrication of photonic devices.

Xiaotao Hao received the Ph.D. degree from Shandong University, Jinan, China, in 2002. He is currently a Professor in the School of Physics, Shandong University, China. He was a Research Associate at the Institute of Materials Research and Engineering, Singapore, from 2002 to 2005. He then moved as a Japan Society for the Promotion of Science fellow to Chiba University, Chiba, Japan. He joined the School of Chemistry, University of Melbourne in 2007 and returned to Shandong University as a Professor in 2012. His research interests include material modifications by ultrafast lasers, time resolved fluorescence spectroscopy/microscopy, organic electronics, etc. He serves as the Regional Vice President of the Thin Films Society. He is a Member of the Material Research Society, Chartered Member of Royal Australian Chemistry Institute, a Member of Asian and Oceanian Photochemistry Association.

Feng Chen received the Ph.D. degree from Shandong University, Jinan, China, in 2002. He is currently a Professor in the School of Physics, Shandong University, Shandong, China. He was with the Clausthal University of Technology, Germany, from 2003 to 2005, as an Alexander von Humboldt Research Fellow. He became a Professor at Shandong University in 2006. His research interests include material modifications by ultrafast lasers and ion beams, optical waveguides, etc. He is a Fellow of the Institute of Physics, U.K., a Senior Member of the Optical Society of America. 